\title{
A Preliminary Study of Inner Hearing in Music Performance
}

\author{
Zhengyu Yan ${ }^{1, *}$ \\ ${ }^{1}$ Academy of Music, Shanxi University, Taiyuan, Shanxi 030006, China \\ *Corresponding author. Email: 290794316@qq.com
}

\begin{abstract}
In music activities, music performance is a bridge between music creation and appreciation. It can show the audience the aesthetic value of musical works. Through performance, musical notes can be turned into emotional music, giving musical works brand new life. Therefore, the music performer is very critical. The performer's hearing is the most important musical feeling, and the value of music is realized by the characteristics of human hearing. This article focuses on piano performance, and the characteristics of hearing correspond to the way it is cultivated in keyboard performance. Through the explanation of the inner hearing of music performance and the importance of inner hearing in the process of technique training and performance, this paper provides some theoretical support for keyboard players in practical skills training and stage performances.
\end{abstract}

Keywords: Auditory characteristics, Inner hearing, Keyboard performance.

\section{INTRODUCTION}

The auditory characteristics of the human ear have different functions such as distinguishing the height, timbre, intensity, loudness, time value, direction, and distance of the sound. The human ear's recognition and perception of these physical properties of sound enables people to integrate objectively generated physical phenomena into the rich psychological world for absorption and processing, and then evolve into emotional experience, which makes music, an objective material product, endowed with rich subjective feelings in the unique spiritual world of human beings.

\section{AUDITORY CHARACTERISTICS}

\subsection{Sense of Timbre}

Timbre is a complex feature that is difficult to explain in words. "The American Standards Association (ASA) proposed that timbre is 'an auditory attribute'. Based on this attribute, the listener can distinguish the difference between two tones with the same pitch and loudness." ${ }^{1}$ In addition to distinguishing pure tones, the human ears can also distinguish compound sounds and harmony of sounds. The acoustic concept of timbre depends on the waveform of the sound wave and its multiplier structure, that is, the number of complex sounds. The concept of timbre formed in the human brain belongs to the category of psychological perception.

The auditory feeling and aesthetics that the timbre gives people are abundant. The high overtone timbre is bright, giving people a bright and pleasant feeling; the low overtone timbre is dark, giving people a dark and deep feeling. The brightness characteristics of timbre can be described as bright, dark, shining, dim, etc.; the texture characteristics of timbre can be described as soft, thick, sharp, blunt, thin, thick, etc.; the spatial characteristics of timbre can be described as full, empty, broad, etc. The rich and colorful timbre features provide a broad space for the performance and aesthetics of music.

In the process of piano performance, the control of timbre is particularly important. The timbre of a

1. (U.S.) Donald A. Hodges, Handbook of Music Psychology [M]. Liu Pei, Ren Kai, trans. Changsha: Hunan Literature and Art Publishing House, 2006, p120. 
work is affected by many different factors. This influence can be divided into two aspects: the timbre outside the work and the timbre in the work.

The timbre outside the work, there is a certain gap in the development level of musical instruments at that time and the environment of aesthetic thoughts of the time, and the development level of musical instruments in different periods. As far as piano development is concerned, the level of piano development in the Beethoven era is completely different from the level of piano development in the Chopin era. As far as the sustain of a note is concerned, the key sustain of a piano in Beethoven's period can reach about seven seconds, but the key sustain of a piano in Chopin's period is about fifteen seconds long. In this state, it is necessary to distinguish between the handling of timbre and the use of pedals when playing. Affected by aesthetic thoughts, the classical period developed from humanism to humanitarianism, focusing on the rational pursuit of artistic forms, and the romantic period developed from rationality to sensibility, the pursuit of the spiritual world. It can be clearly seen from the painting art of the time that the romantic period from indoor painting to outdoor natural light painting. From character paintings to outdoor landscape paintings, people can see the pursuit of nature, sensibility, and spirituality. The same is true in music. Classicism and Romanticism have two different feelings in musical performance. This different musical experience leads people to use different keystrokes in music performance to reflect the meaning of musical performance in different periods given by different timbres.

The timbre in a work cannot have only one timbre effect in a work. A piano piece is like a condensed symphony, in which different parts are played by different instruments. In the keyboard, this requires people to make correct judgments on the relationship between tone and tone, and the relationship between musical zones. Different sound areas and different musical images are distinguished by different timbres. Among them, it is necessary to control the strength of different touch keys, as well as the rapid conversion between different technologies, to achieve proficiency through a lot of practice.

\subsection{Intensity and Sense of Loudness}

"Loudness as a sensory variable is mainly related to the physical characteristic of amplitude. The term volume is often incorrectly used in the discussion of loudness. For example, stereo equipment often has a volume knob to control loudness, but the two are not synonymous. Loudness is related to an increase in intensity, and volume has a complex relationship with intensity, density, and the range of auditory experience. Volume is the psychological perception of the space occupied by sound. For example, when lowfrequency sounds are at high intensity, they seem to be louder than high-frequency sounds at low intensity." 2

The intensity or volume of a sound depends on the amplitude of the sound, which is a physical concept of objective matter. Loudness is a person's subjective feeling and perception of objective sounds, and it also belongs to the category of psychology. The human ear has obvious time value, direction, distance feeling and distinguishing ability. It is the psychological reaction mechanism formed by the human auditory system's acceptance and transmission of sounds produced by objective substances. Time value is the ability of the human ear to compare the duration of continuous sound waves. The sense of distance and direction are the azimuth recognition of the human ear to the transmission of sound waves.

The intensity and loudness of piano performance are relative in the overall musical composition, just like the color palette in painting. There is no absolute blue sky, green grass, and red flowers in the painting. They all determine how blue the sky is, how green the ground is and how red the flowers are compared to the overall tone. The same is true in music, $\mathrm{p}$ is stronger than $\mathrm{pp}$. People can't precisely specify how much power is needed for weak and how much power is given for strong. They only need to use hearing to distinguish the difference between $\mathrm{pp}, \mathrm{p}, \mathrm{mp}$, etc. This requires the training and cultivation of music sensibility.

In addition to the identification of the physical properties of the external sound auditory mechanism, the musical hearing in terms of musical sensibility is equally important, or even more important.

2. (U.S.) Donald A. Hodges, Handbook of Music Psychology [M]. Liu Pei, Ren Kai, trans. Changsha: Hunan Literature and Art Publishing House, 2006, p113. 


\section{CULTIVATION OF MUSIC HEARING IN KEYBOARD PERFORMANCE}

\subsection{Significance of Music Audition Training}

Music art is the art of hearing. Of all the sensations produced by musical activities, the most important sensation is hearing. The aesthetic and performance practice activities of keyboard art mainly rely on the sense of hearing. Appreciation of instrumental music and performance of artistic performance and touch key technology are essentially the art of hearing and the technology of hearing. The extensive participation of auditory physiology and auditory psychology makes performance activities have a clear direction and specific goals, and performance aesthetics and creativity can be maintained and unfolded.

Performance hearing is a musical consciousness activity in which humans exert force on the keys on the keyboard as the specific perceptual object, which belongs to the category of human advanced neural activity and thinking consciousness. Musical hearing plays an important supporting role in performance activities. Musical hearing always affects and restricts the performer's technical behavior in its specific way. For performers, they must truly have a keen musical hearing ability in order to effectively guide their performance practice and effectively improve their performance ability and level.

\subsection{The Subjective and Objective Unity of Keyboard Performance and Hearing}

The performance of keyboard music is a comprehensive mechanism that has both touch key technology and musical artistic expression. As far as the touch key sound technology is concerned, in the performance, because the human finger is in the subject position and the auditory organ is in the object position, a contradiction between subjective and objective will inevitably arise. This kind of contradiction is firstly reflected in the process of touching the keys. Performers must pay attention to the musical emotions and the technical state of the keys, and also rely on the auditory system to adjust the accuracy of the musical aspects of the sound, including timbre, rhythm and other factors. The second is that in the field of performance hearing, it is necessary to rely on the auditory system to adjust the balance of the overall sound effect, but also to rely on the auditory function to adjust the relationship between the midrange and the tone of a musical composition. There is a clear difference between the sound that people feel during performance and the auditory response of others. This is because people's so-called auditory sensations in performance include not only the auditory response that is reflected from the outside world through air conduction, but also the auditory sensation that is conducted from the touch of the finger to the inner ear through the bone and muscle tissue. At the same time, there is a sense of tactile sensation in the body due to sound resonance and vibration. Therefore, this is different from the single auditory experience experienced by the listener in an objective environment. Because of this difference, in keyboard learning and teaching training, there will often be a contradiction between subjective perception and objective evaluation criteria. This requires performers to gradually solve the problem in the teacher's guidance and repeated practice.

\section{INNER HEARING}

Inner hearing ${ }^{3}$ is also known as psychological hearing. This kind of auditory perception always influences and acts on people's performance creation and aesthetic activities. It is a must to form a correct sound concept through the cultivation of inner hearing, to rely on the support of inner hearing to distinguish the difference between subjective hearing and objective sound quality of touch keys, and to master the control of timbre touch keys proficiently and correctly through the guidance of inner hearing to reach the normative art standard, so as to finally complete the goal of musical art performance.

\subsection{The Importance of Inner Hearing in Performance}

Inner hearing promotes the performer's understanding of the beauty of sound. In the field of piano performance, through the effective support of inner hearing, the performers can form a unique cognition of the sound, and better analyze and

3. Inner hearing: Inner hearing is divided into melody and harmony. Melody inner hearing is a kind of melody imagination and recognition ability; harmony inner hearing is a kind of multi-voice, harmony and polyphonic music works imagination and recognition ability. The training of melody inner hearing is relatively quick. Anyone who is engaged in music work usually has this ability. However, the training of harmonic inner hearing is relatively slow, and may not reach the level of proficiency and application. 
explore the aesthetic connotation in the sound, so as to acquire good artistic appreciation ability. In the process of performance, the performers need to feel the connotation of the music from the heart, follow the emotional footsteps of the creator for in-depth appreciation and listening, so as to form a good predictive ability of the music as a whole, and use their own playing skills to perform standardized processing.

In the process of performing specific piano music, the performers can perform effective analysis on the emotional level, feel the emotions that the author wants to express, and adjust and process emotions according to their own true understanding, and then integrate themselves with the real piano performance, so that the whole performance presents a strong appeal and drives the atmosphere of the scene. Under the pillar of inner hearing, performers can also better diverge and imagine thinking, so as to perform better interpretation and expression, so that their artistic level in the field of piano performance can be significantly improved.

\subsection{The Importance of Inner Hearing in Skill Training}

As one of the basic qualities, inner hearing has more prominent value in promoting the further improvement of playing skills. Therefore, in the process of daily participation in piano performance training, performers need to start from the inner level to standardize the specific information related to musical works, and form a strong memory of specific music through processing, storage and other methods, and regulate their participation in piano performance related activities. So, the performers need to attach great importance to the content of piano deduction, and carry out reasonable regulations on specific training methods.

The relationship between inner hearing and piano performance is within the scope of music. As an important project component, piano performance presents more significant artistic appreciation value. In the field of piano performance, the influence exerted by inner hearing is more prominent, and the relationship between the two is relatively close. In the process of playing, if the performers have a good inner auditory ability as a support, they can better appreciate the artistic conception, melody and style of music, and can also gain strong resonance on the emotional level, and then they effectively deal with emotions on this basis, so as to ensure that the piano works they perform have a strong appeal. Therefore, performers need to form an accurate understanding of the relationship between the two, and clarify the importance of inner hearing in the entire piano performing process.

\section{CONCLUSION}

In keyboard learning and training, how to resolve the subjective and objective contradiction of hearing and cultivate the correct musical hearing of performers is a very important physiological, physical and psychological mechanism. Only by mastering the auditory mechanism of music and grasping the law of the unity of subjective and objective contradictions in music learning and training can the ideal effect of music learning and training be achieved. During the performance of piano performance, the inner hearing ability of the performers is an important factor that affects the overall performance. Therefore, it is necessary to form an accurate understanding of the element of inner hearing, to correct the ideological recognition of its importance in piano performance, and to carry out targeted training according to the actual situation. Within the scope of piano performance, it is also necessary to appreciate specific musical works, conduct effective training in terms of emotion, sound, melody, etc., and strengthen the effective construction of one's own imagination control, so as to perform piano works in a better state and improve the comprehensive level of piano performance.

\section{AUTHORS' CONTRIBUTIONS}

This paper is independently completed by Zhengyu Yan.

\section{REFERENCES}

[1] (U.S.) Donald A. Hodges, Handbook of Music Psychology [M]. Liu Pei, Ren Kai, trans. Changsha: Hunan Literature and Art Publishing House, 2006. (in Chinese)

[2] Liang Guangcheng, The Mystery of Musical Sounds [M]. Beijing: People's Music Publishing House, 1986. (in Chinese)

[3] Hei Dun, The Relationship Between Physiology and Psychology and Music [A]. Music Translation Collection, Volume 4 [C]. Beijing: People's Music Publishing House, 1963. (in Chinese) 
[4] Zhou Wen, "Heart-Shaped Unity" in Music Performance: A Probe into the Inner Auditory of Piano Performance [J]. Think Tank Era, 2018(37): 141-142. (in Chinese)

[5] Wang Wei, The Importance of Inner Hearing in Piano Performance and the Ways to Cultivate It [J]. Northern Music, 2019, 39(21): 41-42. (in Chinese)

[6] Lu Lu, Research on Interval Hearing Training in Temporal Hearing [D]. Central Conservatory of Music, 2008. (in Chinese) 